\title{
ON JOINTLY PRIME RADICALS OF (R,S)-MODULES
}

\author{
Dian Ariesta Yuwaningsih ${ }^{1}$ And Indah Emilia Wijayanti ${ }^{2}$ \\ ${ }^{1}$ Postgraduate Student of Mathematics, Universitas Gadjah Mada, \\ Yogyakarta, Indonesia \\ dian.ariesta17@yahoo.com \\ ${ }^{2}$ Department of Mathematics, Universitas Gadjah Mada, \\ Yogyakarta, Indonesia \\ ind_wijayanti@ugm.ac.id
}

\begin{abstract}
Let $M$ be an $(R, S)$-module. In this paper a generalization of the $\mathrm{m}$ system set of modules to $(R, S)$-modules is given. Then for an $(R, S)$-submodule $N$ of $M$, we define $\sqrt[(R, S)]{N}$ as the set of $a \in M$ such that every m-system containing $a$ meets $N$. It is shown that $\sqrt[(R, S)]{N}$ is the intersection of all jointly prime $(R, S)$ submodules of $M$ containing $N$. We define jointly prime radicals of an $(R, S)$-module $M$ as $\operatorname{rad}_{(R, S)}(M)=\sqrt[(R, S)]{0}$. Then we present some properties of jointly prime radicals of an $(R, S)$-module.
\end{abstract}

Key words and Phrases: $(R, S)$-module, jointly prime $(R, S)$-submodule, m-system, prime radical.

\begin{abstract}
Abstrak. Diberikan $(R, S)$-modul $M$. Dalam tulisan ini didefinisikan himpunan sistem-m pada suatu $(R, S)$-modul sebagai perumuman dari himpunan sistemm suatu modul. Didefinisikan $\sqrt[(R, S)]{N}$ sebagai himpunan semua $a \in M$ yang memenuhi sifat setiap sistem-m yang memuat $a$ irisannya dengan $N$ tidak kosong, untuk suatu $(R, S)$-submodul $N$ di $M$. Dapat ditunjukkan bahwa $\sqrt[(R, S)]{N}$ merupakan irisan dari semua $(R, S)$-submodul prima gabungan di $M$ yang memuat $N$. Didefinisikan radikal prima gabungan dari $(R, S)$-modul $M$ sebagai himpunan $\operatorname{rad}_{(R, S)}(M)=\sqrt[(R, S)]{0}$. Kemudian, dalam tulisan ini disajikan beberapa sifat dari radikal prima gabungan suatu $(R, S)$-modul.

Kata kunci: $(R, S)$-modul, $(R, S)$-submodul prima gabungan, sistem-m, radikal prima.
\end{abstract}

2000 Mathematics Subject Classification: 16S90.

Received: 23-05-2013, revised: 01-11-2014, accepted: 24-11-2014. 


\section{Introduction}

All rings in this paper are arbitrary ring unless stated otherwise. Let $R$ and $S$ be arbitrary rings. Khumprapussorn et al. in [3] introduced $(R, S)$-modules as a generalization of $(R, S)$-bimodules. An $(R, S)$-module has an $(R, S)$-bimodule structure when both rings $R$ and $S$ have central idempotent elements.

In their paper, Khumprapussorn et al. also defined $(R, S)$-submodules of $M$ as additive subgroups $N$ of $M$ such that $r n s \in N$ for all $r \in R, n \in N$, and $s \in S$. Moreover, a proper $(R, S)$-submodule $P$ of $M$ is called a jointly prime $(R, S)$ submodule if for each left ideal $I$ of $R$, right ideal $J$ of $S$, and $(R, S)$-submodule $N$ of $M, I N J \subseteq P$ implies $I M J \subseteq P$ or $N \subseteq P$.

A jointly prime $(R, S)$-submodule $P$ of $M$ is called a minimal jointly prime $(R, S)$-submodule if it is minimal in the class of jointly prime $(R, S)$-submodules of $M$. Based on Goodearl and Warfield [2], we show that every jointly prime $(R, S)$-submodule of $M$ contains a minimal jointly prime $(R, S)$-submodule.

Let $T$ be a ring with unity. Lam [4] has defined that a nonempty set $J \subseteq T$ is said to be an m-system if for each pair $a, b \in J$, there exists $t \in T$ such that $a t b \in J$. Furthermore, for an ideal $I$ of $T$, the set $\sqrt{I}:=\{a \in T \mid(\forall$ m-system $J$ of $T) a \in$ $J \Rightarrow J \cap I \neq \emptyset\}$ equals to the intersection of all the prime ideals of $T$ containing $I$. Based on this definition, Behboodi [1] has generalized the definition of m-system of unitary rings to modules. Let $M$ be an unitary module over a ring $T$. A nonempty set $X \subseteq M \backslash\{0\}$ is called an m-system if for each (left) ideal $I$ of $T$ and for all submodules $K, L$ of $M,(K+L) \cap X \neq \emptyset$ and $(K+I M) \cap X \neq \emptyset$ imply $(K+I L) \cap X \neq$ $\emptyset$. It has been shown that the complement of a prime submodule is an m-system, and for any m-system $X$, a submodule disjoint from $X$ and maximal with respect to this property is always a prime submodule. Moreover, for a submodule $N$ of $M$, the set $\sqrt{N}:=\{a \in M \mid(\forall$ m-system $X$ of $M) a \in X \Rightarrow X \cap N \neq \emptyset\}$ equals to the intersection of all prime submodules of $M$ containing $N$.

In Section 2 , we extend these facts to $(R, S)$-modules. In fact, we give a generalization of the notion of m-systems of modules to $(R, S)$-modules. Then for an $(R, S)$-submodule $N$ of $M$, we define $\sqrt[(R, S)]{N}:=\{a \in M \mid(\forall$ m-system $X$ of $M) a \in$ $X \Rightarrow X \cap N \neq \emptyset\}$. And then we define jointly prime radicals of an $(R, S)$-module $M$ as $\operatorname{rad}_{(R, S)}(M)=\sqrt[(R, S)]{0}$. It is shown that $\operatorname{rad}_{(R, S)}(M)$ is the intersection of all jointly prime $(R, S)$-submodules of $M$ (note that, if $M$ has no any jointly prime $(R, S)$-submodule, then $\left.\operatorname{rad}_{(R, S)}(M):=M\right)$. In Section 3, we present some properties of jointly prime radicals of $(R, S)$-modules. These properties are as follows: every jointly prime radicals of $(R, S)$-submodules is contained in a jointly prime radical of its $(R, S)$-module; jointly prime radicals of $(R, S)$-modules $M$ is either equal to $M$ or the intersection of all minimal jointly prime $(R, S)$-submodules of $M$; and jointly prime radicals of quotient $(R, S)$-modules $M / \operatorname{rad}_{(R, S)}(M)$ is zero. 


\section{Jointly Prime Radicals of (R,S)-Modules}

Before we define m-systems of an $(R, S)$-module, we describe first the jointly prime $(R, S)$-submodule. As we have already stated earlier, a proper $(R, S)$ submodule $P$ of $M$ is called a jointly prime $(R, S)$-submodule if for each left ideal $I$ of $R$, right ideal $J$ of $S$, and $(R, S)$-submodule $N$ of $M, I N J \subseteq P$ implies $I M J \subseteq P$ or $N \subseteq P$. The following are some characterizations of jointly prime $(R, S)$-submodules given in [3].

Theorem 2.1. Let $M$ be an $(R, S)$-module satisfying $a \in R a S$ for all $a \in M$, and $P$ a proper $(R, S)$-submodule of $M$. The following statements are equivalent:

(1) $P$ is a jointly prime $(R, S)$-submodule.

(2) For every right ideal $I$ of $R, m \in M$, and left ideal $J$ of $S$, ImJ $\subseteq P$ implies $I M J \subseteq P$ or $m \in P$.

(3) For every right ideal $I$ of $R,(R, S)$-submodule $N$ of $M$, and left ideal $J$ of $S, I N J \subseteq P$ implies $I M J \subseteq P$ or $N \subseteq P$.

(4) For every left ideal $I$ of $R, m \in M$, and right ideal $J$ of $S,(I R) m(S J) \subseteq P$ implies $I M J \subseteq P$ or $m \in P$.

(5) For every $a \in R, m \in M$, and $b \in S,(a R) m(S b) \subseteq P$ implies $a M b \subseteq P$ or $m \in P$.

If the $(R, S)$-module $M$ satisfies $M=R M S$, the necessary and sufficient condition for a proper $(R, S)$-submodule $P$ of $M$ to be a jointly prime $(R, S)$ submodule is for all ideal $I$ of $R$, ideal $J$ of $S$, and $(R, S)$-submodule $N$ of $M$, $I N J \subseteq P$ implies $I M J \subseteq P$ or $N \subseteq P$.

Now, we define the notion of m-systems of $(R, S)$-modules.

Definition 2.2. Let $M$ be an $(R, S)$-module. A nonempty set $X \subseteq M \backslash\{0\}$ is called an $m$-system if for each left ideal $I$ of $R$, right ideal $J$ of $S$, and $(R, S)$-submodules $K, L$ of $M,(K+L) \cap X \neq \emptyset$ and $(K+I M J) \cap X \neq \emptyset$ imply $(K+I L J) \cap X \neq \emptyset$.

Based on Behboodi [1], we can show that the complement of a jointly prime $(R, S)$-submodule is an m-system.

Proposition 2.3. Let $P$ be a proper $(R, S)$-submodule of $M$. Then $P$ is a jointly prime $(R, S)$-submodule of $M$ if and only if $X=M \backslash P$ is an m-system.

Proof. $(\Rightarrow)$. Suppose that $P$ is a jointly prime $(R, S)$-submodule of $M$. Let $I$ be a left ideal of $R, J$ be a right ideal of $S$, and $K, L$ be $(R, S)$-submodules of $M$ such that $(K+L) \cap X \neq \emptyset$ and $(K+I M J) \cap X \neq \emptyset$. If $(K+I L J) \cap X=\emptyset$, then $K+I L J \subseteq P$. Then, $I L J \subseteq P$ and $K \subseteq P$. Since $P$ is a jointly prime $(R, S)$-submodule of $M$, we have $L \subseteq P$ or $I M J \subseteq P$. Thus $(K+L) \cap X=\emptyset$ or $(K+I M J) \cap X=\emptyset$, a contradiction. Therefore, $X$ is an m-system of $M$.

$(\Leftarrow)$. Suppose that $X$ is an m-system of $M$. Let $I$ be a left ideal of $R, J$ be a right ideal of $S$, and $L$ be an $(R, S)$-submodule of $M$ such that $I L J \subseteq P$. If $L \nsubseteq P$ and $I M J \nsubseteq P$, then $L \cap X \neq \emptyset$ and $I M J \cap X \neq \emptyset$. Since $X$ is an m-system, $I L J \cap X \neq \emptyset$ so that $I L J \nsubseteq P$, a contradiction. Therefore, $P$ is a jointly prime $(R, S)$-submodule of $M$. 
Example 2.4. Let $\mathbb{Z}$ be the ring of integers taken as an $(2 \mathbb{Z}, 3 \mathbb{Z})$-module. First, we show that $6 \mathbb{Z}$ is a jointly prime $(2 \mathbb{Z}, 3 \mathbb{Z})$-submodule of $\mathbb{Z}$. Consider a left ideal $I=(2 m) \mathbb{Z}$ of $2 \mathbb{Z}$, a right ideal $J=(3 n) \mathbb{Z}$ of $3 \mathbb{Z}$, and an $(2 \mathbb{Z}, 3 \mathbb{Z})$-submodule

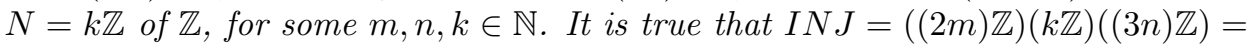
$(6 m k n) \mathbb{Z} \subseteq 6 \mathbb{Z}$ and $N=k \mathbb{Z}=\nsubseteq \subseteq 6 \mathbb{Z}$. Then for each $m, n \in \mathbb{N}$, it is clear that $I \mathbb{Z} J=((2 m) \mathbb{Z})(\mathbb{Z})((3 n) \mathbb{Z})=(6 m n) \mathbb{Z} \subseteq 6 \mathbb{Z}$. Hence, $6 \mathbb{Z}$ is a jointly prime $(2 \mathbb{Z}, 3 \mathbb{Z})$ submodule of $\mathbb{Z}$. Therefore, $\mathbb{Z} \backslash 6 \mathbb{Z}$ is an m-system of $(2 \mathbb{Z}, 3 \mathbb{Z})$-module $\mathbb{Z}$.

It is easy to prove that every maximal $(R, S)$-submodule of $M$ is a jointly prime $(R, S)$-submodule. Furthermore, we prove a proposition that states that a maximal $(R, S)$-submodule $P$ of $M$ which is disjoint from an arbitrary m-system of $M$ is a jointly prime $(R, S)$-submodule.

Proposition 2.5. Let $M$ be an $(R, S)$-module, $X$ an m-system of $M$, and $P$ a proper $(R, S)$-submodule of $M$ maximal with respect to the property that $P \cap X=\emptyset$. Then, $P$ is a jointly prime $(R, S)$-submodule of $M$.

Proof. Let $I$ be a left ideal of $R, J$ a right ideal of $S$, and $N$ an $(R, S)$-submodule of $M$ such that $I N J \subseteq P$. Suppose that $N \nsubseteq P$ and $I M J \nsubseteq P$. Since $P$ is maximal with respect to the property that $P \cap X=\emptyset$, we have $(P+N) \cap X \neq \emptyset$ and $(P+I M J) \cap X \neq \emptyset$. Since $X$ is an m-system of $M$, then $(P+I N J) \cap X \neq \emptyset$. Since $I N J \subseteq P$, it follows that $P \cap X \neq \emptyset$, a contradiction. Therefore, $P$ must be a jointly prime $(R, S)$-submodule of $M$.

We recall the set introduced by Behboodi in [1],

$$
\sqrt{N}:=\{a \in M \mid(\forall \text { m-system } X \text { of } M) a \in X \Rightarrow X \cap N \neq \emptyset\} .
$$

Now, we present a generalization of the notion of $\sqrt{N}$ for any $(R, S)$-submodules $N$ of $M$ and we denote it as $\sqrt[(R, S)]{N}$.

Definition 2.6. Let $M$ be an $(R, S)$-module. For an $(R, S)$-submodule $N$ of $M$, if there is a jointly prime $(R, S)$-submodule containing $N$, then we define $\sqrt[(R, S)]{N}:=$ $\{a \in M \mid(\forall$ m-system $X$ of $M) a \in X \Rightarrow X \cap N \neq \emptyset\}$. If there is no jointly prime $(R, S)$-submodules containing $N$, then we define $\sqrt[(R, S)]{N}:=M$.

Let $M$ be an $(R, S)$-module. Then, the jointly prime spectrum of $M$ is the set $\operatorname{Spec}^{j_{p}}(M):=\{P \mid P$ is a jointly prime $(R, S)$-submodule of $M\}$. If $N$ be an $(R, S)$-submodule of $M$, then we define $V^{j_{p}}(N):=\left\{P \in \operatorname{Spec}^{j_{p}}(M) \mid N \subseteq P\right\}$. Next, we show that $\sqrt[(R, S)]{N}$ equals to the intersection of all jointly prime $(R, S)$ submodules of $M$.

Theorem 2.7. Let $M$ be an $(R, S)$-module and $N$ be an $(R, S)$-submodule of $M$. Then either $\sqrt[(R, S)]{N}=M$ or $\sqrt[(R, S)]{N}=\bigcap_{P \in V^{j_{p}}(N)} P$.

Proof. Suppose that $\sqrt[(R, S)]{N} \neq M$. It follows from Definition 2.6 that $V^{j_{p}}(N) \neq \emptyset$. We will show that $\sqrt[(R, S)]{N}=\bigcap_{P \in V^{j_{p}}(N)} P$. Let $m \in \sqrt[(R, S)]{N}$ and $P \in V^{j_{p}}(N)$. 
Consider the m-system $X:=M \backslash P$ in $M$. Since $N \subseteq P$, we have $X \cap N=\emptyset$. Consequently, we get $m \notin X$ so that $m \in P$. Thus, we obtain $\sqrt[(R, S)]{N} \subseteq \bigcap_{P \in V^{j_{p}}(N)} P$. Conversely, let $a \in \bigcap_{P \in V^{j_{p}}(N)} P$. If $a \notin \sqrt[(R, S)]{N}$, then there exists an m-system $X$ such that $a \in X$ but $N \cap X=\emptyset$. Consider the following set:

$$
\mathfrak{J}=\{J \mid N \subseteq J, J \text { is an }(R, S) \text {-submodule of } M \text { and } J \cap X=\emptyset\} .
$$

By Zorn's Lemma, $\mathfrak{J}$ has a maximal element, which is an $(R, S)$-submodule $K \supseteq N$ maximal with respect to the property $K \cap X=\emptyset$. By Proposition 2.5, $K$ is a jointly prime $(R, S)$-submodule of $M$, so $K \in V^{j_{p}}(N)$. Therefore, we have $a \in K$. Whereas $a \in X$, so we get $K \cap X \neq \emptyset$, a contradiction. Thus, $a \in \sqrt[(R, S)]{N}$ and it follows that $\bigcap_{P \in V^{j_{p}}(N)} P \subseteq \sqrt[(R, S)]{N}$. Hence, $\sqrt[(R, S)]{N}=\bigcap_{P \in V^{j_{p}}(N)} P$.

Example 2.8. Let $\mathbb{Z}$ be an $(2 \mathbb{Z}, 2 \mathbb{Z})$-module and $8 \mathbb{Z}$ be an $(2 \mathbb{Z}, 2 \mathbb{Z})$-submodule of $\mathbb{Z}$. We obtain the set $V^{j_{p}}(8 \mathbb{Z})=\left\{P \in\right.$ Spec $\left.^{j_{p}}(\mathbb{Z}) \mid 8 \mathbb{Z} \subseteq P\right\}=\{2 \mathbb{Z}, 4 \mathbb{Z}\}$. Therefore, $\sqrt[(2 \mathbb{Z}, 2 \mathbb{Z})]{8 \mathbb{Z}}=\bigcap_{P \in V^{j_{p}}(8 \mathbb{Z})} P=4 \mathbb{Z} \cap 2 \mathbb{Z}=4 \mathbb{Z}$.

Let $I$ be an ideal of an unitary ring $T$. By Lam [4], $\sqrt{I}$ is equal to $T$ or the intersection of all prime ideals of $T$ containing $I$. From Khumprapussorn et al. [3], we know that the annihilator from $M / N$ of the ring $R$, that is $(N: M)_{R}:=\{r \in$ $R \mid r M S \subseteq N\}$, is an ideal of $R$ when the ring $S$ satisfies $S^{2}=S$. Therefore, when $S^{2}=S, \sqrt{(N: M)_{R}}$ is equal to $R$ or the intersection of all prime ideals of $R$ containing $(N: M)_{R}$. Next, we present a connection between $\sqrt{(N: M)_{R}} M S$ and $\sqrt[(R, S)]{N}$

Proposition 2.9. Let $M$ be an $(R, S)$-module and $N$ be an $(R, S)$-submodule of $M$. If $S^{2}=S$, then $\sqrt{(N: M)_{R}} M S \subseteq \sqrt[(R, S)]{N}$.

Proof. Since $S^{2}=S$, by [3] $(N: M)_{R}$ is an ideal of $R$. Also $\sqrt{(N: M)_{R}}$ is equal to $R$ or equal to the intersection of all prime ideals of $R$ that contain $(N: M)_{R}$.

Suppose that $\sqrt[(R, S)]{N}=M$. Since $\sqrt{(N: M)_{R}} \subseteq R$, so

$$
\sqrt{(N: M)_{R}} M S \subseteq R M S \subseteq M=\sqrt[(R, S)]{N} .
$$

Suppose that $\sqrt[(R, S)]{N} \neq M$. Then $\sqrt[(R, S)]{N}=\bigcap_{P \in V^{j_{p}}(N)} P$. Let $P \in V^{j_{p}}(N)$, then $P$ is a jointly prime $(R, S)$-submodule of $M$ and $N \subseteq P$. Moreover, by Proposition 2.12 of [3], $(P: M)_{R}$ is a prime ideal of $R$. Furthermore, since $N \subseteq P$, it is clear that $(N: M)_{R} \subseteq(P: M)_{R}$. Since $(P: M)_{R}$ is a prime ideal of $R$ and contains $(N: M)_{R}$, we obtain

Thus,

$$
\sqrt{(N: M)_{R}} \subseteq(P: M)_{R}
$$

$$
\sqrt{(N: M)_{R}} M S \subseteq(P: M)_{R} M S \subseteq P .
$$


Therefore, this shows that $\sqrt{(N: M)_{R}} M S \subseteq \bigcap_{P \in V^{j p}(N)} P=\sqrt[(R, S)]{N}$.

The definition of jointly prime radicals of an $(R, S)$-module is given below.

Definition 2.10. Let $M$ be an $(R, S)$-module. If there is a jointly prime $(R, S)$ submodule of $M$, then we define jointly prime radicals of $M$ as:

$$
\operatorname{rad}_{(R, S)}(M)=\sqrt[(R, S)]{0}:=\bigcap_{P \in \operatorname{Spec}^{j_{p}}(M)} P .
$$

If there is no jointly prime $(R, S)$-submodule of $M$, then we define jointly prime radicals of $M$ as $\operatorname{rad}_{(R, S)}(M):=M$.

Example 2.11. Let $\mathbb{Z}$ be an $(2 \mathbb{Z}, 2 \mathbb{Z})$-module. It is easy to show that $\{0\}$ is a jointly prime $(2 \mathbb{Z}, 2 \mathbb{Z})$-submodule of $\mathbb{Z}$. Since every jointly prime $(2 \mathbb{Z}, 2 \mathbb{Z})$-submodule of $\mathbb{Z}$ contains $\{0\}$, then jointly prime radical of $(2 \mathbb{Z}, 2 \mathbb{Z})$-module $\mathbb{Z}$ is $\operatorname{rad}_{(2 \mathbb{Z}, 2 \mathbb{Z})}(\mathbb{Z})=\{0\}$.

\section{Some Properties of Jointly Prime Radicals of $(R, S)$-Modules}

In this section, we present some properties of jointly prime radicals of $(R, S)$ modules. Let $N$ be an $(R, S)$-submodule of $M$. We show that the jointly prime radical of $N$ is contained in the jointly prime radical of $M$.

Proposition 3.1. Let $N$ be an $(R, S)$-submodule of $M$. Then, $\operatorname{rad}_{(R, S)}(N) \subseteq$ $\operatorname{rad}_{(R, S)}(M)$.

Proof. Let $P \in \operatorname{Spec}^{j_{p}}(M)$. If $N \subseteq P$ then $\operatorname{rad}_{(R, S)}(N) \subseteq P$. If $N \nsubseteq P$ then it is easy to check that $N \cap P$ is a jointly prime $(R, S)$-submodule of $N$, and hence $\operatorname{rad}_{(R, S)}(N) \subseteq N \cap P \subseteq P$. So, in any case we get $\operatorname{rad}_{(R, S)}(N) \subseteq P$. Thus, it follows that $\operatorname{rad}_{(R, S)}(N) \subseteq \operatorname{rad}_{(R, S)}(M)$.

In module theory, we know that if $T$-module $M$ is a direct sum of its submodules then the prime radicals of $M$ is also a direct sum of prime radicals of its submodules. Evidently, this property is still maintained on $(R, S)$-modules $M$ when $M$ satisfies $a \in R a S$ for all $a \in M$.

Proposition 3.2. Let $M$ be an $(R, S)$-module and $\left\{N_{i}\right\}_{i \in I}$ be a collection of $(R, S)$ submodules of $M$. If $M$ satisfies $a \in R a S$ for all $a \in M$ and $M=\bigoplus_{i \in I} N_{i}$ then we have $\operatorname{rad}_{(R, S)}(M)=\bigoplus_{i \in I} \operatorname{rad}_{(R, S)}\left(N_{i}\right)$.

Proof. Since each $N_{i}$ is an $(R, S)$-submodule of $M$, we get $\operatorname{rad}_{(R, S)}\left(N_{i}\right) \subseteq$ $\operatorname{rad}_{(R, S)}(M)$ for each $i \in I$. Thus, it follows that

$$
\bigoplus_{i \in I} \operatorname{rad}_{(R, S)}\left(N_{i}\right) \subseteq \operatorname{rad}_{(R, S)}(M) .
$$


Now, let $m \in M$. Then, $m=\sum_{i \in I} m_{i}$ with $m_{i} \in N_{i}$ for each $i \in I$ and $m_{i}=0$ except for finitely many indices $i \in I$. Suppose that $m \notin \bigoplus_{i \in I} \operatorname{rad}_{(R, S)}\left(N_{i}\right)$. We will prove that $m \notin \operatorname{rad}_{(R, S)}(M)$. Since $m \notin \bigoplus_{i \in I} \operatorname{rad}_{(R, S)}\left(N_{i}\right)$, then there exists $k \in I$ such that $m_{k} \notin \operatorname{rad}_{(R, S)}\left(N_{k}\right)$. Thus, there exists a jointly prime $(R, S)$-submodule $N_{k}^{*}$ of $N_{k}$ such that $m_{k} \notin N_{k}^{*}$. Consider $K=N_{k}^{*} \bigoplus\left(\bigoplus_{i \neq k} N_{i}\right)$. First, we prove that $K$ is a jointly prime $(R, S)$-submodule of $M$. Let $I$ be a right ideal of $R, J$ be a left ideal of $S$, and $a \in M$ such that $I a J \subseteq K$. Since $M$ satisfies $a \in R a S$ for all $a \in M$, then based on Theorem 2.1 we will prove that $I M J \subseteq K$ or $a \in K$. Since $a \in M, a=\sum_{i \in I} a_{i}$ where $a_{i} \in N_{i}$ for each $i \in I$ and $a_{i}=0$ except for finitely many indices $i \in I$. Thus we get $I a J=I\left(\sum_{i \in I} a_{i}\right) J=I a_{k} J+I\left(\sum_{i \neq k} a_{i}\right) J \subseteq K$, so that $I a_{k} J \subseteq N_{k}^{*}$. Since $N_{k}^{*}$ is a jointly prime $(R, S)$-submodule of $N_{k}$, we have $I N_{k} J \subseteq N_{k}^{*}$ or $a_{k} \in N_{k}^{*}$. Since $a_{i} \in N_{i}$ for each $i \in I, \sum_{i \neq k} a_{i} \in \bigoplus_{i \neq k} N_{i}$. Since for all $i \in I, N_{i}$ is an $(R, S)$-submodule of $M, I\left(\bigoplus_{i \neq k} N_{i}\right) J \subseteq \bigoplus_{i \neq k} N_{i}$. Thus, it follows that $a=\sum_{i \in I} a_{i} \in K$ or $I\left(\bigoplus_{i \in I} N_{i}\right) J=I M J \subseteq K$. Hence, $K$ is a jointly prime $(R, S)$-submodule of $M$. Furthermore, because $m_{k} \notin N_{k}^{*}$ then $m \notin K$. Since $K$ is a jointly prime $(R, S)$-submodule of $M, m \notin \operatorname{rad}_{(R, S)}(M)$. Thus, it follows that

$$
\operatorname{rad}_{(R, S)}(M) \subseteq \bigoplus_{i \in I} \operatorname{rad}_{(R, S)}\left(N_{i}\right) .
$$

From (1) and (2), we obtain $\operatorname{rad}_{(R, S)}(M)=\bigoplus_{i \in I} \operatorname{rad}_{(R, S)}\left(N_{i}\right)$.

It is easy to show that every jointly prime $(R, S)$-submodule of $M$ contains a minimal jointly prime $(R, S)$-submodule of $M$. Based on this property, we get a relationship between jointly prime radicals of $(R, S)$-modules and minimal jointly prime $(R, S)$-submodules.

Proposition 3.3. Let $M$ be an $(R, S)$-module. The jointly prime radical of $M$ is equal to $M$ or the intersection of all minimal jointly prime $(R, S)$-submodules of $M$.

Proof. Since every jointly prime $(R, S)$-submodule of $M$ contains a minimal jointly prime $(R, S)$-submodule then for each $P \in \operatorname{Spec}^{j_{p}}(M)$ there exists a minimal jointly prime $(R, S)$-submodule $P^{\prime} \in \operatorname{Spec}^{j_{p}}(M)$ such that $P^{\prime} \subseteq P$. Furthermore, we can form the set:

$$
\Im=\left\{P^{\prime} \mid P^{\prime} \text { is a minimal jointly prime }(R, S) \text {-submodule }\right\} .
$$

Suppose that $\operatorname{rad}_{(R, S)}(M) \neq M$. We will prove that $\operatorname{rad}_{(R, S)}(M)=\bigcap_{P^{\prime} \in \Im} P^{\prime}$. Since $\Im \subseteq \operatorname{Spec}^{j_{p}}(M)$, we get $\operatorname{rad}_{(R, S)}(M) \subseteq \bigcap_{P^{\prime} \in \Im} P^{\prime}$. On the other hand, for any 
$P \in \operatorname{Spec}^{j_{p}}(M)$ there is $P^{*} \in \Im$ with $P^{*} \subseteq P$. Thus $\bigcap_{P^{\prime} \in \Im} P^{\prime} \subseteq P^{*} \subseteq P$, which implies that $\bigcap_{P^{\prime} \in \Im} P^{\prime} \subseteq \operatorname{rad}_{(R, S)}(M)$. Hence $\operatorname{rad}_{(R, S)}(M)=\bigcap_{P^{\prime} \in \Im} P^{\prime}$. Therefore, this shows that $\operatorname{rad}_{(R, S)}(M)$ is equal to the intersection of all minimal jointly prime $(R, S)$-submodules of $M$.

Now, we give an important lemma which will be used in the proof of the next property of jointly prime radicals of an $(R, S)$-module.

Lemma 3.4. Let $P_{1}$ and $P_{2}$ be jointly prime $(R, S)$-submodules of $M$, and let $P_{1} / \operatorname{rad}_{(R, S)}(M)$ and $P_{2} / \operatorname{rad}_{(R, S)}(M)$ be $(R, S)$-submodules of $M / \operatorname{rad}_{(R, S)}(M)$. Then,

$$
P_{1} / \operatorname{rad}_{(R, S)}(M)^{\cap} P_{2} / \operatorname{rad}_{(R, S)}(M)=\left(P_{1} \cap P_{2}\right) / \operatorname{rad}_{(R, S)}(M) .
$$

Given an $(R, S)$-module $M$ and $(R, S)$-submodules $A, P$ of $M$ with $A \subset P$. Then, it is easy to check that the necessary and sufficient condition for $P$ to be a jointly prime $(R, S)$-submodule of $M$ is $P / A$ being a jointly prime $(R, S)$-submodule of $M / A$. By using this property, we can show that the jointly prime radical of the quotient $(R, S)$-module $M / \operatorname{rad}_{(R, S)}(M)$ is zero.

Proposition 3.5. Let $M$ be an $(R, S)$-module. Then,

$$
\operatorname{rad}_{(R, S)}\left(M / \operatorname{rad}_{(R, S)}(M)\right)=\overline{0} .
$$

Proof. Suppose that $M$ has no jointly prime $(R, S)$-submodules, then we get that quotient $(R, S)$-modules $M / \operatorname{rad}_{(R, S)}(M)$ also has no jointly prime $(R, S)$ submodules. Thus, $\operatorname{rad}_{(R, S)}(M)=M$ and then we obtain

$$
\operatorname{rad}_{(R, S)}\left(M / \operatorname{rad}_{(R, S)}(M)\right)=\operatorname{rad}_{(R, S)}(M / M)=\operatorname{rad}_{(R, S)}(\overline{0})=\overline{0} .
$$

Suppose that $M$ has a jointly prime $(R, S)$-submodule, then we obtain that quotient $(R, S)$-module $M / \operatorname{rad}_{(R, S)}(M)$ also has a jointly prime $(R, S)$-submodule. From the definition,

$$
\operatorname{rad}_{(R, S)}\left(M / \operatorname{rad}_{(R, S)}(M)\right)=\bigcap_{\bar{P} \in \operatorname{Spec}^{j_{p}}\left(M / \operatorname{rad}_{(R, S)}(M)\right)} \bar{P} .
$$

Since Lemma 3.4 can be generalized for infinite number of $P_{i}$ jointly prime $(R, S)$ submodules of $M$, then we get

$$
\bigcap_{\bar{P} \in \operatorname{Spec}^{j_{p}}\left(M / \operatorname{rad}_{(R, S)}(M)\right)} \bar{P}=\left(\bigcap_{P \in \operatorname{Spec}^{j_{p}}(M)} P\right) / \operatorname{rad}_{(R, S)}(M) .
$$


So,

$$
\operatorname{rad}_{(R, S)}\left(M / \operatorname{rad}_{(R, S)}(M)\right)=\operatorname{rad}_{(R, S)}(M) / \operatorname{rad}_{(R, S)}(M)=\overline{0} .
$$

Hence, it's proved that $\operatorname{rad}_{T}\left(M / \operatorname{rad}_{T}(M)\right)=\overline{0}$.

Given an $(R, S)$-module $M$ and an ideal $I$ of $R$ such that $I \subseteq A n n_{R}(M)$. We can show that an $(R, S)$-module $M$ is also an $(R / I, S)$-module under the scalar multiplication operation that defined as follows:

$$
\begin{aligned}
{ }_{-} \cdot_{-}: R / I \times M \times S & \longrightarrow M \\
(\bar{a}, m, s) & \longrightarrow \bar{a} \cdot m \cdot s:=a m s
\end{aligned}
$$

for all $\bar{a} \in R / I, m \in M$, and $s \in S$.

Moreover, it is easy to check that $P$ is a jointly prime $(R, S)$-submodule of $M$ if and only if $P$ is a jointly prime $(R / I, S)$-submodule of $M$.

Proposition 3.6. Let $M$ be an $(R, S)$-module and $I$ be an ideal of $R$ such that $I \subseteq \operatorname{Ann}_{R}(M)$. Then, $\operatorname{rad}_{(R, S)}(M)=\operatorname{rad}_{(R / I, S)}(M)$.

Proof. Let $a \in \operatorname{rad}_{(R, S)}(M)$ and $P$ be a jointly prime $(R, S)$-submodule of $M$. Then, $a \in P$. Since $P$ is also a jointly prime $(R / I, S)$-submodule of $M$, $a \in \operatorname{rad}_{(R / I, S)}(M)$. Thus, we obtain

$$
\operatorname{rad}_{(R, S)}(M) \subseteq \operatorname{rad}_{(R / I, S)}(M) .
$$

Furthermore, let $b \in \operatorname{rad}_{(R / I, S)}(M)$ and $N$ a jointly prime $(R / I, S)$-submodule of $M$. Then, $b \in N$. Since $N$ is also a jointly prime $(R, S)$-submodule of $M$, $b \in \operatorname{rad}_{(R, S)}(M)$. Thus, we get

$$
\operatorname{rad}_{(R / I, S)}(M) \subseteq \operatorname{rad}_{(R, S)}(M) .
$$

Based on (3) and (4), it's proved that $\operatorname{rad}_{(R, S)}(M)=\operatorname{rad}_{(R / I, S)}(M)$.

\section{Concluding Remarks}

Further work on the properties of jointly prime radicals of an $(R, S)$-module can be carried out. For example, the investigation of properties of jointly prime radicals can be done on any left multiplication $(R, S)$-module. The concept of left multiplication $(R, S)$-modules has been described by Khumprapussorn et al. [3].

Acknowledgement This work is a part of first author's thesis. We are thankful to the referees for the useful comments and feedbacks. 


\section{REFERENCES}

[1] Behboodi, M., "On the Prime Radical and Baer's Lower Nilradical of Modules", Acta Mathematica Hungarica, 122 (2009), 293-306.

[2] Goodearl, K., R. and Warfield, R.B., An Introduction to Noncommutative Noetherian Rings, Cambridge University Press, 2004.

[3] Khumprapussorn, T., Pianskool, S., and Hall, M., (R,S)-Modules and their Fully and Jointly Prime Submodules, International Mathematical Forum, 7 (2012), 1631-1643.

[4] Lam, T.Y., A First Course in Noncommutative Rings, Springer-Verlag New York, Inc., 2001. 